\title{
Newly acquired Onchocerca volvulus filariae after doxycycline treatment
}

\author{
Sabine Specht • Achim Hoerauf • Ohene Adjei • \\ Alexander Debrah • Dietrich W. Büttner
}

Received: 2 June 2009 / Accepted: 27 August 2009 /Published online: 16 September 2009

(C) The Author(s) 2009. This article is published with open access at Springerlink.com

\begin{abstract}
Despite successful mass drug administration and vector control programs, the nematode Onchocerca volvulus is far from being eradicated. Therefore, new long-term sterilizing or macrofilaricidal drugs are needed. The depletion of Wolbachia endobacteria using doxycycline leads to long-term sterilizing effects and macrofilaricidal activity against female filariae of more than $60 \%$. The worms die or degenerate 18-27 months after doxycycline. However, during this time patients may be exposed to new infections. We evaluated these newly acquired worms in onchocercomas of doxycycline-treated patients in relation to transmission using morphology, histochemistry, and immunohistology. On an average, $10 \%$ of the female filariae had been newly acquired per year in the treated groups. Our observations showed: (a) Sixty-three of 68 newly acquired worms harbored many intact Wolbachia, whereas none of the other surviving worms contained many
\end{abstract}

\author{
S. Specht $(\bowtie) \cdot$ A. Hoerauf \\ Institute for Medical Microbiology, \\ Immunology and Parasitology, University Hospital Bonn, \\ Sigmund-Freud-Str. 25, \\ 53105 Bonn, Germany \\ e-mail: specht@microbiology-bonn.de \\ O. Adjei $\cdot$ A. Debrah \\ Kumasi Centre for Collaborative Research in Tropical Medicine \\ (KCCR), \\ Kumasi, Ghana \\ O. Adjei $\cdot$ A. Debrah \\ School of Medical Sciences, \\ Kwame Nkrumah University of Science and Technology, \\ Kumasi, Ghana \\ D. W. Büttner \\ Bernhard Nocht Institute for Tropical Medicine, \\ Bernhard-Nocht-Str. 74, \\ 20359 Hamburg, Germany
}

bacteria. (b) Higher percentages of dead filariae than originally reported were calculated, when the new worms were excluded, indicating a stronger macrofilaricidal activity than previously reported. The difference was significant for female filariae after doxycycline treatment for 6 weeks. (c) Only newly acquired worms presented normal embryogenesis and microfilariae production after sufficient treatment. We conclude that newly acquired filariae have to be considered when evaluating drug efficacy in onchocerciasis.
Abbreviations
APR O. volvulus aspartic protease
IVM ivermectin
L3 infective larvae
mf microfilaria (e)
WSP Wolbachia surface protein

\section{Introduction}

Onchocerciasis can lead to devastating skin disease and blindness. Ivermectin (IVM, Mectizan ${ }^{\circledR}$ ) distribution within the African Programme for Onchocerciasis Control (APOC) had a marked impact on the disease, reducing prevalence of blindness and transmission of onchocerciasis. Recent surveys done by APOC indicate that approximately 37 million people may still be infected (Basañez et al. 2006), and modeling studies suggest that IVM treatments alone may not lead to onchocerciasis elimination (Alley et al. 2001). This and the risk of emergence of IVM-resistant parasites necessitate the search for alternative drugs or drug combinations, preferably with macrofilaricidal or long- 
lasting sterilizing effects or a complementary vaccine (Duke 2002).

Clinical trials require optimal methods for the evaluation of drug activity, since for ethical and economical reasons reliable information should be gained from as few patients with as little risk as possible. Usually, onchocerciasis trials are carried out in endemic areas with still ongoing transmission. This implies that patients are exposed and new infections will be acquired. To evaluate the efficacy of a drug or vaccine, it is necessary to differentiate between worms present at the time of drug or vaccine administration and worms acquired during long observation periods. Such long periods are needed for the detection of long-term sterilizing or slow macrofilaricidal effects.

An example for such long observation periods is the treatment with antibiotics. Onchocerca volvulus and other filariae contain Wolbachia bacteria, which are essential endosymbionts for parasite fertility and survival. Several clinical trials with doxycycline have already been completed. The first study demonstrated that a six-week treatment with $100 \mathrm{mg}$ doxycycline per day leads to the elimination of intact Wolbachia after 6-12 months and to long-term sterilization (Hoerauf et al. 2003). After a longer observation of 2027 months, up to $60 \%$ of the female filariae were found dead in two recent studies (Hoerauf et al. 2008, 2009). Since these studies were performed in an area with ongoing transmission, a proportion of the worms that were still living after these longer observation periods must have been newly acquired.

Consequently, their subtraction from the number of all observed worms will change the figures previously reported for the efficacy of doxycycline. We have described the morphological and immunohistological criteria for the diagnosis of newly acquired worms in a previous report (Specht et al. 2009). Here, we assess the number of newly acquired young worms in doxycycline trials using these criteria. Our hypotheses for this study were: (a) Twelve or more months after doxycycline all $O$. volvulus filariae with many not degenerated Wolbachia were worms newly acquired after treatment. (b) The exclusion of the worms newly acquired after doxycycline leads to lower percentages of living worms and hence to higher percentages of dead filariae indicating a stronger macrofilaricidal efficacy. (c) Eighteen or more months after doxycycline all worms with normal embryogenesis and microfilariae $(\mathrm{mf})$ production have been acquired after the treatment.

\section{Patients, material, and methods}

Study area

The results presented here are mainly based on two doxycycline trials performed between 2003 and 2006
(Hoerauf et al. 2008, 2009). In addition, data were also considered from the first trial (Hoerauf et al. 2003) and from further dose-finding trials in 2000-2002 (unpublished data). Patients for these studies had been selected in several villages in the Central Region of Ghana. In 1999, this area was found to be hyperendemic for onchocerciasis during rapid assessment of 30 men older than 19 years per village in Upper Denkyira and Assin districts, with $\mathrm{mf}$ carrier rates between $47 \%$ and $90 \%$ of the examined men (R. Horstmann and D.W. Büttner). In most of the villages, IVM treatment had been administered beginning in 1999, but a reduction of the infectivity rates and the loads of infective larvae (L3) in Simulium sanctipauli was not observed in the trial villages between 2002 and 2006 (Kutin et al. 2004; R. Garms, Bernhard Nocht Institute, unpublished report, 2006). Transmission was ongoing during the trials.

\section{Treatment of patients}

Patients were treated with 100 or $200 \mathrm{mg}$ doxycycline per day for 4, 5, or 6 weeks. Most patients took a single dose of IVM a few months after doxycycline. Control patients received matching placebos or remained untreated, except for IVM. Onchocercomas were extirpated aseptically under local anesthesia in the regional hospital in Dunkwa. Patients were nodulectomized at various follow-up time points after the start of treatment, ranging from 2-39 months (Hoerauf et al. 2003, 2008, 2009).

\section{Immunohistology}

Onchocercomas were fixed in $70-80 \%$ ethanol or $4 \%$ phosphate-buffered formaldehyde solution and embedded in paraffin using standard methods. Hematoxylin eosin (Merck, Darmstadt, Germany) was used for all nodules. For the detection of iron in the filarial tissues, Gomori's iron method was applied for all nodules selected for this study, using acid potassium ferrocyanide solution and nuclear fast red as counter stain (Luna 1968). For immunohistology, the alkaline phosphatase antialkaline phosphatase technique was applied according to the recommendations given by the manufacturer (DakoCytomation, Hamburg, Germany). An antiserum raised against lysosomal aspartic protease (APR) in a rabbit (Jolodar et al. 2004, supplied by PD Dr Norbert Brattig, Bernhard Nocht Institute) was used as primary antibody to assess vitality and to estimate worm age, diluted 1:1,000-1:4,000. To demonstrate the presence of Wolbachia, a rabbit antiserum against Dirofilaria immitis Wolbachia surface protein (WSP, Bazzocchi et al. 2000, supplied by Prof. Claudio Bandi, Milano, Italy) was used, diluted 1:200-1:4,000. As secondary antibody, mouse antirabbit immunoglobulin (clone MR12/53, Dako- 
Cytomation) was applied. Fast Red TR salt (Sigma, Deisenhofen, Germany) was used as chromogen and hematoxylin (Merck) served as the counter stain. For the electron microscopic (Fig. 1 (6)) showing degenerated Wolbachia, standard methods were used as described previously (Pfarr et al. 2008).

Onchocercomas were analyzed for morphological and immunohistological characteristics of newly acquired young worms. All filariae were examined for vitality, productivity, presence of Wolbachia, and the human nodule tissue was screened for mf. Morphological criteria for the estimation of the worm age and vitality have been described (Büttner et al. 1988; Franz 1988; Schulz-Key 1988; Duke 1991 and in detail by Specht et al. 2009). The classification of female $O$. volvulus follows Duke et al. (2002) with minor modifications. Two authors (DWB and SS) examined the sections independently. When the examiners did not agree, slides were reexamined, which mostly led to a consensus. In doubtful cases, the worms were not counted as newly acquired.

\section{Statistics}

The paired $t$ test was applied to test differences between previously observed and newly calculated rates of dead filariae, using GraphPad ${ }^{\circledR}$ Prism Software.

\section{Results}

Number of newly acquired filariae

Here, we report the results of our reexamination of the onchocercomas from two recent doxycycline trials (Hoerauf et al. 2008, 2009) using the criteria for the differentiation between young and old $O$. volvulus published previously (Specht et al. 2009). Tables 1 and 2 present the numbers and percentages of living young worms identified as newly acquired in different patient groups: a total of 68 female and 32 male filariae. The number of newly acquired males (Table 2) was probably slightly underestimated, because in nodule sections with only few male worm sections not all distinct criteria for the age estimation could be applied and some male worms near to the edge of the nodule may have been completely missed. In the treatment groups, on average $10 \%$ (median $9.0 \%$ ) of the female and $9 \%$ (median $6.4 \%$ ) of the male filariae were identified per year as newly acquired after doxycycline treatment. In the untreated groups on average, only $7 \%$ females and $5 \%$ male worms were identified as newly acquired per year, because the diagnosis was more difficult for untreated filariae.
Elimination of Wolbachia by doxycycline treatment

The elimination of Wolbachia by doxycycline is the precondition for sterilization and death of the filariae. Using immunohistology and PCR, we have shown that most filariae lose their endobacteria during the months after sufficient doxycycline treatment (Hoerauf et al. 2003, 2008, 2009). However, always small numbers of worms with many morphologically intact endobacteria were observed after 20-27 months and this raised the question whether they had been doxycycline-treated or were acquired only afterwards.

The number of bacteria in immature worms is limited, as it is in L3, and therefore we only saw endobacteria in three of seven immature worms (Fig. 1), which had been newly acquired during the last year before nodulectomy (according to the definition of these worms being immature). More than 19 months after doxycycline 23 nulliparous worms were identified as newly acquired. They were all Wolbachia-positive (Table 1, Figs. 1 (2) and (3)). During the months after, doxycycline bacteria started to degenerate in the hypodermis and then in the oocytes. Therefore, a few worms harbored small numbers of endobacteria in degenerated oocytes 11 months after treatment, but these bacteria were obviously degenerated (compare Figs. 1 (4) and (5)). The degenerated bacteria showed an irregular cytoplasm with electron dense inclusions (Fig. 1 (6)) compared to living Wolbachia (e.g., Fig. 1 in Pfarr et al. 2008). Eleven and more months after sufficient doxycycline treatment, the Wolbachia-positive male worms showed the criteria of young worms (Figs. 1 (7) and (8)) when compared to adjacent old female worms. Especially the low iron content of these young worms was striking (Fig. 1 (8)). All 17 productive female worms identified as newly acquired after doxycycline contained many morphologically intact endobacteria (Table 1, Figs. 2 (9), (10), (11) and (14)), except one worm with few bacteria. In contrast, several young fecund females were observed during the first 6 months of observation (Table 1 in Hoerauf et al. 2003) and these young as well as old females contained no Wolbachia since they all had been acquired before treatment

\section{Macrofilaricidal activity of doxycycline}

Secondly, we hypothesized that taking new worms into account, this would affect the macrofilaricidal activity of doxycycline. We subtracted the numbers of newly acquired worms from those of all observed worms and recalculated new percentages of dead worms using the new figures of all worms. In all groups of untreated and doxycycline-treated patients, the corrected percentages of dead female filariae were higher than the original figures, since these included the newly acquired filariae (Table 2). The difference was 

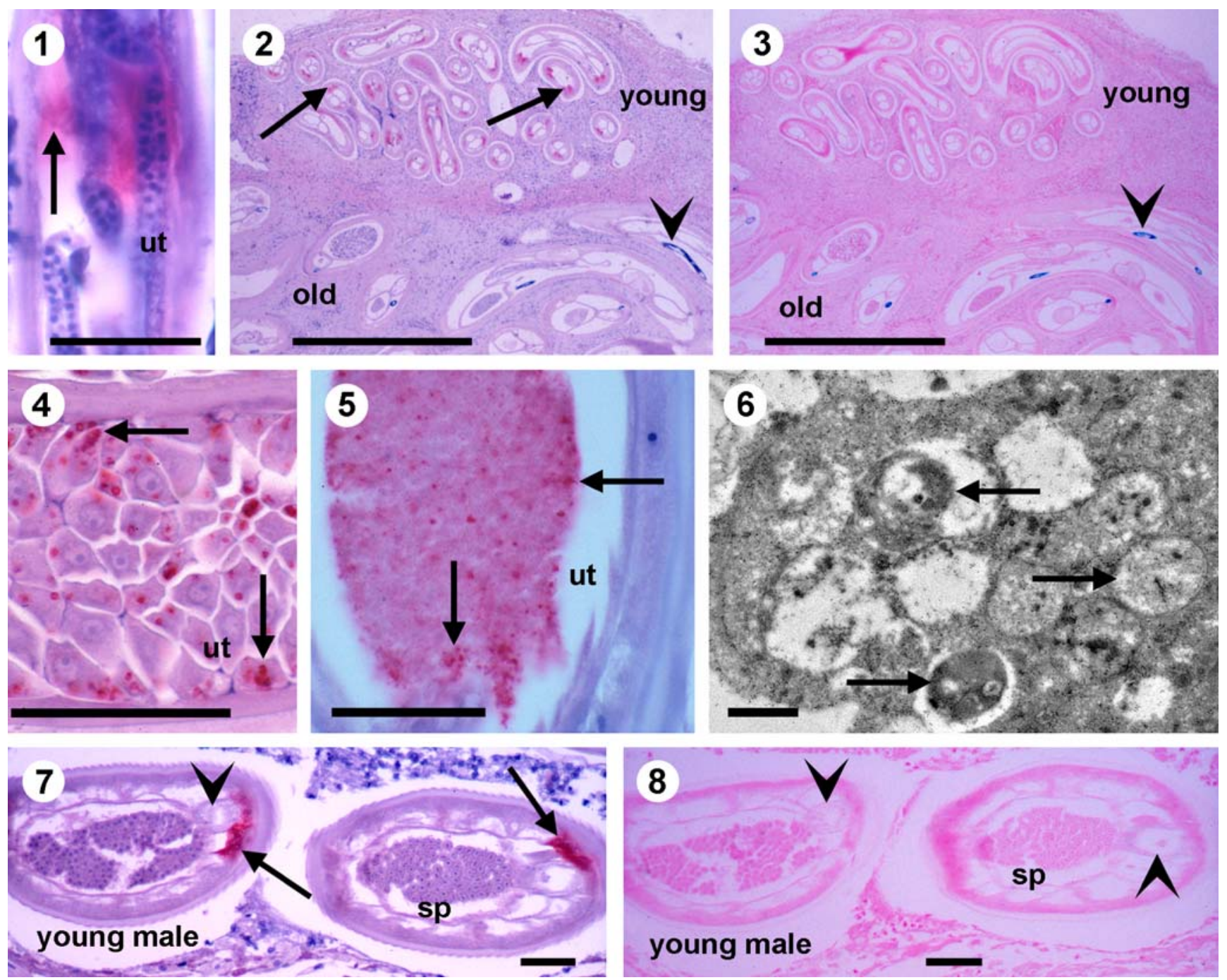

Fig. 11 A Wolbachia-positive (arrow) immature female $O$. volvulus, excised 20 months after $100 \mathrm{mg}$ doxycycline daily for 5 weeks, was certainly newly acquired. The uterus $(u t)$ is small and empty. WSP, scale bar $=50 \mu \mathrm{m}$. 2, 3 A nulliparous young Wolbachia-positive (arrows) female filaria with no iron in the gut was certainly newly acquired in contrast to the bacteria-negative, doxycycline-treated old female with much iron in the gut (arrowheads). Excised 11 months after $100 \mathrm{mg}$ doxycycline for 6 weeks. 2 WSP. 3 Iron stain, scale bar= $50 \mu \mathrm{m}$. 4 Living endobacteria (arrows) in intact oocytes in the uterus $(u t)$ of an untreated worm. WSP, scale bar $=50 \mu \mathrm{m}$. 5 Degenerated endobacteria (arrows) in degenerated oocytes in the uterus $(u t)$ of a doxycycline-treated female worm. Excised 11 months after $100 \mathrm{mg}$

doxycycline for 6 weeks in contrast to the intact endobacteria in 4 . WSP, scale bar $=50 \mu \mathrm{m}$. 6 Electron micrograph of an oocyte with degenerated Wolbachia (arrows), which show irregular cytoplasm and electron dense inclusions. Excised 11 months after $100 \mathrm{mg}$ doxycycline for 6 weeks. Scale bar $=1 \mu \mathrm{m}$. 7 A Wolbachia-positive (arrows) newly acquired young male worm with intact spermatogenesis $(s p)$, contains no iron in the gut (arrowheads). Excised 27 months after $200 \mathrm{mg}$ doxycycline for 6 weeks, WSP, iron stain, scale bar $=50 \mathrm{~mm}$. 8 Wolbachia-positive (arrows) newly acquired young male worm with intact spermatogenesis $(s p)$, contains no iron in the gut (arrowheads). Excised 27 months after $200 \mathrm{mg}$ doxycycline for 6 weeks, WSP, iron stain, scale bar $=50 \mathrm{~mm}$

significant for the female worms treated 6 weeks $(p=$ 0.050). The corrected percentages were also higher for males except for the three groups with $0 \%$. Combining all doxycycline-treated groups examined after 20-39 months into one group, the difference between the previous and the corrected percentages was significant for the female filariae ( $p=0.0018$, paired $t$ test). For the male worms, the difference showed only a trend for higher rates of dead worms, but it was not significant $(p=0.073$ for all and $p=$

0.094 for 6 weeks). Hence, the macrofilaricidal activity of doxycycline was higher in the previous studies than originally reported (Hoerauf et al. 2008, 2009).

Cessation of embryogenesis and $\mathrm{mf}$ production

In our previous reports, we had observed that a small number of filariae presented still normal embryogenesis and $\mathrm{mf}$ were found in the human nodule issue and the skin up to 
Table 1 Embryogenesis and presence of Wolbachia in newly acquired $O$. volvulus

\begin{tabular}{|c|c|c|c|c|c|c|c|c|}
\hline \multirow{3}{*}{$\begin{array}{l}\text { Treatment } \\
\text { group }\end{array}$} & \multirow{3}{*}{$\begin{array}{l}\text { Months after } \\
\text { study onset }\end{array}$} & \multirow{3}{*}{$\begin{array}{l}\text { Number of } \\
\text { patients/nodules }\end{array}$} & \multicolumn{6}{|c|}{ Number of living female worms } \\
\hline & & & \multicolumn{4}{|c|}{ Newly acquired } & \multirow{2}{*}{$\begin{array}{l}\text { Number of } \\
\text { newly acquired }\end{array}$} & \multirow{2}{*}{$\begin{array}{l}\text { Number containing } \\
\text { many Wolbachia }\end{array}$} \\
\hline & & & All & Immature & $\begin{array}{l}\text { Oocytes } \\
\text { only }\end{array}$ & $\begin{array}{l}\text { Embryos/ } \\
\mathrm{mf}\end{array}$ & & \\
\hline \multirow[t]{3}{*}{ Placebo $^{\mathrm{a}}$} & 6 & $13 / 14$ & 29 & 0 & 1 & 0 & 1 & 1 \\
\hline & 20 & $16 / 51$ & 70 & 1 & 4 & 6 & 11 & 10 \\
\hline & 27 & $7 / 38$ & 61 & 0 & 3 & 2 & 5 & 5 \\
\hline \multirow{3}{*}{$\begin{array}{l}\text { Doxycycline }^{\mathrm{a}, \mathrm{b}} \\
4 \text { weeks }\end{array}$} & 20 & $7 / 36$ & 27 & 0 & 1 & 1 & 2 & 2 \\
\hline & 27 & $7 / 23$ & 20 & 0 & 4 & 2 & 6 & 6 \\
\hline & 39 & $2 / 13$ & 12 & 0 & 1 & 1 & 2 & 2 \\
\hline \multirow{4}{*}{$\begin{array}{l}\text { Doxycycline }^{\mathrm{a}, \mathrm{b}} \\
6 \text { weeks }\end{array}$} & 6 & $11 / 19$ & 32 & 0 & 0 & 1 & 1 & 0 \\
\hline & 20 & $13 / 62$ & 42 & 3 & 5 & 3 & 11 & 9 \\
\hline & 27 & $5 / 27$ & 15 & 1 & 3 & 1 & 5 & 5 \\
\hline & 39 & $2 / 7$ & 9 & 0 & 2 & 2 & 4 & 4 \\
\hline Not treated ${ }^{\mathrm{c}}$ & 27 & $10 / 41$ & 51 & 1 & 5 & 0 & 6 & 5 \\
\hline \multirow{2}{*}{$\begin{array}{l}\text { Doxycycline }^{c} \\
5 \text { weeks }\end{array}$} & 20 & $12 / 49$ & 34 & 1 & 2 & 2 & 5 & 4 \\
\hline & 27 & $13 / 47$ & 49 & 0 & 5 & 4 & 9 & 9 \\
\hline
\end{tabular}

${ }^{\mathrm{a}}$ Hoerauf et al. (2008)

b 39 months after treatment: unpublished data

${ }^{\mathrm{c}}$ Hoerauf et al. (2009)

39 months after doxycycline (Hoerauf et al. 2008, 2009). Our hypothesis was that these productive worms had not been treated since they had been newly acquired after doxycycline. Because of the long prepatency, newly acquired worms need a period of one to several years to produce mf. We observed 24 productive new females among the newly acquired worms (Table 1; Fig. 2 (14)). One of them was doubtful since it was seen already after 6 months. But we found no old productive worms after sufficient doxycycline treatment for 5 or 6 weeks (see Table 4 in Hoerauf et al. 2008 and Table 3, 2009). Twenty to 27 months after sufficient doxycycline treatment, only in those few nodules containing newly acquired Wolbachiapositive productive worms numerous bacteria-positive $\mathrm{mf}$ were found adjacent to these female worms (Figs. 2 (14) and (15)). Since we did not detect Wolbachia-positive $\mathrm{mf}$ later than 6 months after doxycycline in nodules without new females, we assume that $\mathrm{mf}$ was only produced by new females, and the sterilization of the female worms present at the time of doxycycline treatment was complete.

\section{Discussion}

To fully evaluate macrofilaricidal drug efficacy in human onchocerciasis, worms present at the time of treatment have to be differentiated from those acquired afterwards, because longer trial periods of a few years need to be adhered to, during which the treated individuals are still being exposed to L3. In the present study, we used morphological, histochemical, and immunohistological criteria for the diagnosis of potentially newly acquired worms from older ones in doxycycline treated patients. Doxycycline leads to long-term sterilization by elimination of Wolbachia endosymbionts (Hoerauf et al. 2003, 2008).

At 18-39 months, no endobacteria were found in worms that met the criteria of older worms and must therefore have already been present during treatment. We have no evidence that there is any recovery of endobacterial populations in worms arising from a few bacteria that we may not have detected. But this can only be definitely proven with a longer observation until all sufficiently treated filariae have died. In contrast, all worms that were described at that time point as young, including fecund ones, contained many morphologically intact Wolbachia. We have to admit that scanning for worms with many bacteria during histological examination has facilitated the detection of newly acquired worms in the doxycycline treatment groups as the higher percentages show. However, all of them were confirmed as young worms based on other criteria than the endobacteria.

A slightly but significantly higher macrofilaricidal activity than originally reported was observed after doxycycline treatment, when newly acquired worms were considered. 


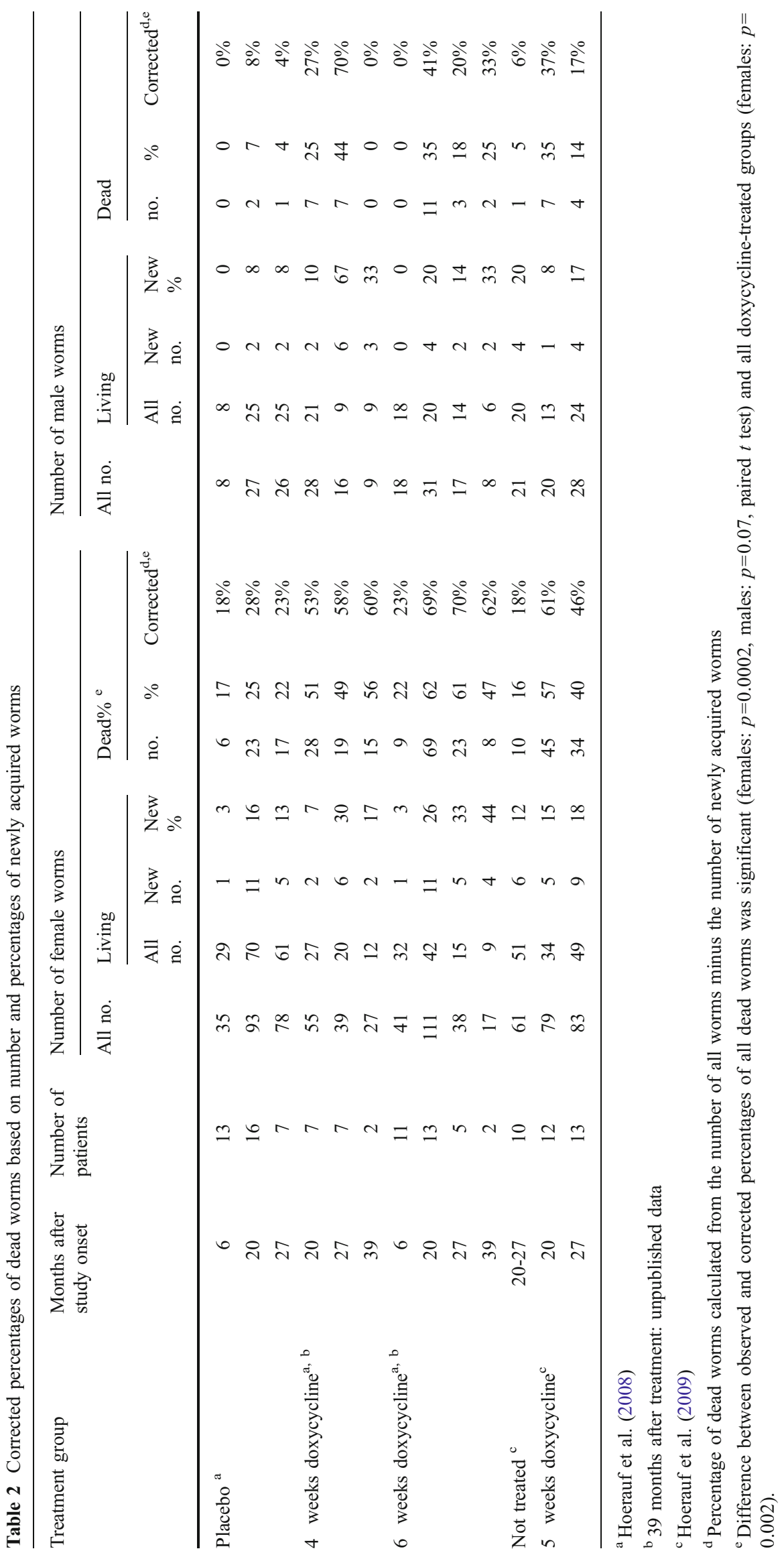



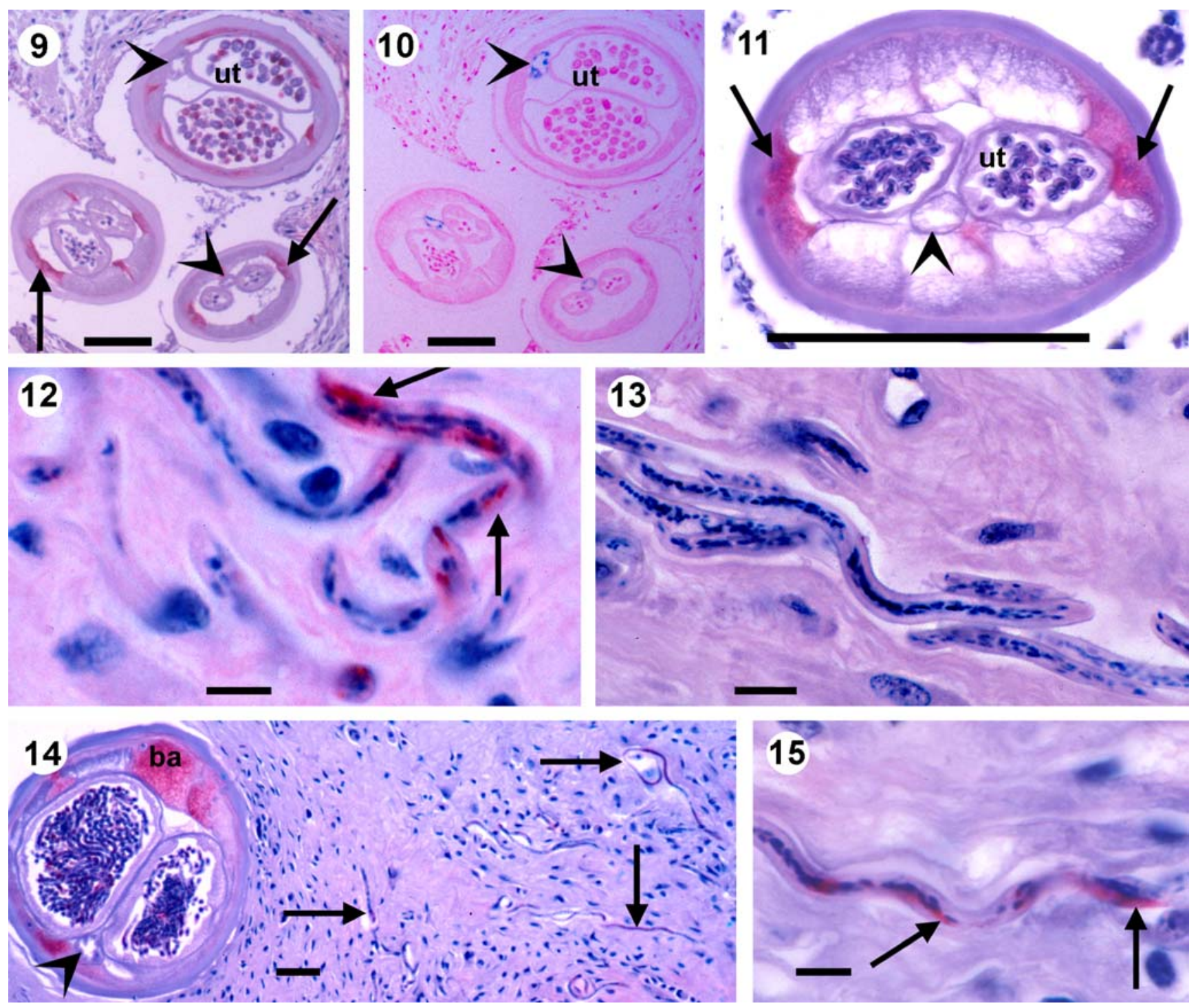

Fig. 2 A newly acquired young female worm full of endobacteriapositive morulae in the uterus $(u t)$ and with sparsely iron in the gut (arrowheads). Excised 27 months after $200 \mathrm{mg}$ doxycycline daily for 6 weeks. 9 WSP, 10 iron stain, scale bar $=100 \mu \mathrm{m}$. 11 A newly acquired young productive female worm with mature $\mathrm{mf}$ in the uterus (ut) and a light gut (arrowhead) contains Wolbachia (arrows). Excised 27 months after $100 \mathrm{mg}$ doxycycline for 5 weeks. WSP, scale bar= $100 \mu \mathrm{m}$. 12 A Wolbachia-positive $\mathrm{mf}$ in a nodule from an untreated patient. WSP, scale bar $=10 \mu \mathrm{m} .13$ Two months after $100 \mathrm{mg}$ doxycycline daily for 6 weeks are still $\mathrm{mf}$ in the nodule but they no longer harbor endobacteria. WSP, scale bar $=10 \mu \mathrm{m}$. 14 Adjacent to a newly acquired, young, endobacteria-positive $(b a)$ female worm with a light gut (arrowhead) are many Wolbachia-positive mf (arrows). Excised 27 months after $100 \mathrm{mg}$ doxycycline for 5 weeks. WSP, scale bar $=50 \mu \mathrm{m}$. 15 Detail of a Wolbachia-positive $\mathrm{mf}$ from 14. WSP, scale bar $=10 \mu \mathrm{m}$
Still, not all worms present during doxycycline treatment were dead after 18-39 months. This observation period might therefore not be long enough for all filariae to die. We believe that they will die later as degenerated filariae after long years of vector control do (Albiez et al. 1987; Büttner et al. 1990). However, we cannot formally exclude that a few of them may recover in spite of their severe degeneration. On the other hand, survival of a small number of worms is accepted for other anthelmintic drugs, e.g., for schistosomes (WHO 1995).
Embryogenesis and $\mathrm{mf}$ production were interrupted by doxycycline treatment. It was clearly visible that 1139 months after treatment, all Wolbachia-positive mf were found adjacent to an endobacteria-harboring female with the characteristics of a newly acquired worm and all these $\mathrm{mf}$ were bacteria-positive. We assume that all the endobacteria-positive $\mathrm{mf}$ seen 18 months and later had been produced by newly acquired filariae. The life expectancy of $\mathrm{mf}$ varies, but on average 18 months is assumed (Plaisier et al. 1991). Probably, none or only few 
doxycycline-treated mf survived after 20 and more months. This is probably also true for the $\mathrm{mf}$ in the skin (Hoerauf et al. 2008, 2009). The results confirm a long-term sterilizing effect of doxycycline. Since the treated surviving females had either degenerated oocytes or empty uteri, the sterilization is probably permanent. Further, these severely degenerated filariae will probably die and not recover.

We assume that $9-10 \%$ of the living worms observed at the follow-up examinations had been newly acquired annually since the transmission was ongoing in the trial villages (Kutin et al. 2004; R. Garms, Bernhard Nocht Institute, unpublished report, 2006). This percentage is sufficient to replenish the number of worms dying naturally, which may be about $10 \%$ each year, if we assume an average life expectancy of 10 years. Plaisier et al. (1991) report 9-11 years as average life expectancy in different villages in the savannah areas. We estimated similar figures based on the decline of worms in the upper part of the body in children (Büttner and Weiss 1984) and after vector control in Burkina Faso and Ghana (Büttner et al. 1988, 1990). In Burkina Faso, we nodulectomized patients after interruption of transmission in 1977, 1978, 1980, 1983, and 1987 and analyzed their worm loads, which decreased continually. After these 11 years, the burden of living worms per person was only $10 \%$ of that in 1977 and some of these worms had obviously been acquired in neighboring Ivory Coast (Albiez et al. 1987). Our assumption that about $10 \%$ of worms die annually is based on these figures. The approximately $10 \%$ of the adult worm population, which die each year in chronic infections, will more or less be replenished. Schulz-Key and Soboslay (2000) found that such stable parasite loads are maintained in communities with longstanding immunity despite the different intensities of transmission. Thus differing proportions of transmitted L3 will develop into adult worms, varying between less than $0.1 \%$ and $10 \%$ or more, whereas most L3 are eliminated by the host. Stable $O$. volvulus loads in highly endemic foci had been shown to be an important mechanism for parasite density regulation by Duerr et al. (2003). These findings support our present results that within 23 years roughly $20-30 \%$ of the adult filariae are newly acquired worms.

\section{Conclusions}

We found our three hypotheses confirmed. (a) All the 61 adult and two of the immature of the 68 newly acquired filariae harbored many intact endobacteria, whereas after 12 and more months of doxycycline treatment, none of the other surviving worms harbored many endobacteria. (b) Higher percentages of dead filariae than originally reported were calculated, when the newly acquired worms were excluded; therefore, the real macrofilaricidal activity of doxycycline is higher than originally reported. This difference was significant for the group of female filariae after sufficient doxycycline treatment for 6 weeks. (c) Only worms identified as newly acquired presented normal embryogenesis and $\mathrm{mf}$ production, and all $\mathrm{mf}$ observed in the nodule tissue harbored Wolbachia 18 and more months after sufficient doxycycline treatment. We conclude further that newly acquired filariae have to be considered for proper evaluation of drug efficacy in onchocerciasis.

Acknowledgements We thank Ingeborg Albrecht, Christel Schmetz, and Klaus Jürries for technical assistance. We are obliged to Prof. Claudio Bandi (Milano, Italy) for the antiWSP serum, PD Dr. Norbert Brattig for the APR serum, and Dr. Kenneth Pfarr for critical reading of the manuscript. We are grateful for financial support from the European Commission (EU-grants ICA4-CT-2002-10051 and INCOCT-2006-032321).

Ethical approval The doxycycline trials have been approved by the Ethical Committee of the Medical Board Hamburg or the Liverpool School of Tropical Medicine and the Ethical Committee of the School of Medical Sciences of the Kwame Nkrumah University of Science and Technology, Kumasi, Ghana (Hoerauf et al. 2003, 2008, 2009). Study procedures were in accordance with the Declaration of Helsinki (1975 and its revisions in 1983, 2000, and 2002). The trial was registered at Current Control Trials (registration number 71141922).

Open Access This article is distributed under the terms of the Creative Commons Attribution Noncommercial License which permits any noncommercial use, distribution, and reproduction in any medium, provided the original author(s) and source are credited.

\section{References}

Albiez EJ, Kaiser A, Büttner DW (1987) The worm burden of Onchocerca volvulus in patients in Burkina Faso twelve years after interruption of transmission. Trop Med Parasitol 38:348 abstract only

Alley WS, van Oortmarssen GG, Boatin BB, Nagelkerke NN, Plaisier AA, Remme HJ, Lazdins J, Borsboom GJ, Habbema JD (2001) Macrofilaricides and onchocerciasis control, mathematical modelling of the prospects for elimination. BMC Public Health 1:12

Basañez MG, Pion SD, Churcher TS, Breitling LP, Little MP, Boussinesq M (2006) River blindness: a success story under threat? PLoS Med 3:e371

Bazzocchi C, Ceciliani F, McCall JW, Ricci I, Genchi C, Bandi C (2000) Antigenic role of the endosymbionts of filarial nematodes: IgG response against the Wolbachia surface protein in cats infected with Dirofilaria immitis. Proc R Soc Lond B Biol Sci 267:2511-2516

Büttner DW, Weiss IJ (1984) The macrofilaria burden of children with onchocerciasis in West Africa. Proc Int Congr Infect Dis Wien $153-156$

Büttner DW, Albiez EJ, von Essen J, Erichsen J (1988) Histological examination of adult Onchocerca volvulus and comparison with the collagenase technique. Trop Med Parasitol 39:390-417 
Büttner DW, Awadzi K, Opoku NO (1990) Histological studies of onchocercomata from an area with interrupted transmission in Ghana. Acta Leiden 59:49-50

Duerr HP, Dietz K, Schulz-Key H, Büttner DW, Eichner M (2003) Density-dependent parasite establishment suggests infectionassociated immunosuppression as an important mechanism for parasite density regulation in onchocerciasis. Trans $\mathrm{R}$ Soc Trop Med Hyg 97:242-250

Duke BOL (1991) Observations and reflections on the immature stages of Onchocerca volvulus in the human host. Ann Trop Med Parasitol 85:103-110

Duke BOL (2002) A plea to continue the search for an Onchocerca volvulus macrofilaricide. Trans R Soc Trop Med Hyg 96:575-576

Duke BOL, Marty AM, Peett DL, Gardo J, Pion SD, Kamgno J, Boussinesq M (2002) Neoplastic change in Onchocerca volvulus and its relation to ivermectin treatment. Parasitology 125:431-444

Franz M (1988) The morphology of adult Onchocerca volvulus based on electron microscopy. Trop Med Parasitol 39:359-366

Hoerauf A, Mand S, Volkmann L, Büttner M, Marfo-Debrekyei Y, Taylor M, Adjei O, Büttner DW (2003) Doxycycline in the treatment of human onchocerciasis: kinetics of Wolbachia endobacteria reduction and of inhibition of embryogenesis in female Onchocerca worms. Microbes Infect 5:261-273

Hoerauf A, Specht S, Büttner M, Pfarr K, Mand S, Fimmers R, MarfoDebrekyei Y, Konadu P, Debrah AY, Bandi C, Brattig N, Albers A, Larbi J, Batsa L, Taylor MJ, Adjei O, Büttner DW (2008) Wolbachia endobacteria depletion by doxycycline as antifilarial therapy has macrofilaricidal activity in onchocerciasis: a randomized placebo-controlled study. Med Microbiol Immunol 197:295-311

Hoerauf A, Specht S, Marfo-Debrekyei Y, Büttner M, Debrah AY, Mand S, Batsa L, Brattig N, Konadu P, Bandi C, Fimmers R,
Adjei O, Büttner DW (2009) Efficacy of 5-week doxycycline treatment on adult Onchocerca volvulus. Parasitol Res 104:437447

Jolodar A, Fischer P, Büttner DW, Miller DJ, Schmetz C, Brattig NW (2004) Onchocerca volvulus: expression and immunolocalization of a nematode cathepsin D-like lysosomal aspartic protease. Exp Parasitol 107:145-156

Kutin K, Kruppa TF, Brenya R, Garms R (2004) Efficiency of Simulium sanctipauli as a vector of Onchocerca volvulus in the forest zone of Ghana. Med Vet Entomol 18:167-173

Luna LG (1968) Manual of histologic staining methods of the Armed Forces Institute of Pathology, 3rd edn. McGraw-Hill Book Company, New York

Pfarr KM, Heider U, Schmetz C, Büttner DW, Hoerauf A (2008) The mitochondrial heat shock protein 60 (HSP60) is up-regulated in Onchocerca volvulus after the depletion of Wolbachia. Parasitology 135:529-538

Plaisier AP, van Oortmarssen GJ, Remme J, Habbema JD (1991) The reproductive lifespan of Onchocerca volvulus in West African savanna. Acta Trop 48:271-284

Schulz-Key H (1988) The collagenase technique: how to isolate and examine adult Onchocerca volvulus for the evaluation of drug effects. Trop Med Parasitol 39:423-440

Schulz-Key H, Soboslay PT (2000) Parasitological and epidemiological aspects of the host-parasite interaction in filariases and their relevance for intervention and control. Nova Acta Leopold 313:189-204

Specht S, Brattig N, Büttner M, Büttner DW (2009) Criteria for the differentiation between young and old Onchocerca volvulus filariae. Parasitol Res (in press)

WHO (1995) Drugs used in parasitic diseases, 2nd edn. WHO, Geneva 